\title{
A Cultural Experience to support Mental Health in People Aged 16-24 During COVID-19 Compared to a Typical Museum Website: Study Protocol of an Online Randomised Controlled Trial
}

\section{Rebecca Syed Sheriff ( $\nabla$ rebecca.sheriff@psych.ox.ac.uk)}

University of Oxford https://orcid.org/0000-0002-5934-6722

\section{Matti Vuorre}

Oxford Internet Institute, University of Oxford

\section{Evgenia Riga}

University of Oxford, Department of Psychiatry

\section{Andrew K Przybylski}

Oxford Internet Institute, University of Oxford

\section{Helen Adams}

Gardens, Libraries and Museums Division, Ashmolean Museum, University of Oxford

Catherine J Harmer

Department of Psychiatry, University of Oxford

John R Geddes

Department of Psychiatry, University of Oxford

\section{Research Article}

Keywords: Depression, Anxiety, Youth, Experimental Medicine

Posted Date: February 9th, 2021

DOI: https://doi.org/10.21203/rs.3.rs-181910/v1

License: (c) (1) This work is licensed under a Creative Commons Attribution 4.0 International License. Read Full License

Version of Record: A version of this preprint was published at Trials on July 22nd, 2021. See the published version at https://doi.org/10.1186/s13063-021-05441-z. 


\section{Abstract}

Background: Despite the high prevalence of common mental disorders in adolescents and young adults, and their association with poor health and socio-economic outcomes throughout the lifespan, many young people do not seek or receive help for such disorders. There is growing interest in the community sector in supporting mental health in young people, however, there is little by way of experimental research in this area. During the COVID-19 pandemic and lockdown we designed an online cultural experience to reduce anxiety and depression and promote positive mental health in people aged 16-24.

Methods/design: The O-ACE POP (Online Active Community Engagement Proof of Principle) study is a randomised controlled trial of an online cultural experience named Ways of Being, based on human centred narratives and viewpoints, compared with a typical museum website (the Ashmolean Museum). We aim to compare efficacy on mood, distress (depression and anxiety), flourishing and loneliness as well as investigating potential mechanisms of action, and feasibility of a larger scale RCT.

Discussion: COVID-19 has provided a unique opportunity to design an innovative approach to supporting mental health in young adults. Findings derived from this study will allow us to evaluate the efficacy of this intervention and will inform the design of studies to further refine the resource and test it further.

\section{Trial Status}

Trial Registration: NCT04663594

First Registered: $11^{\text {th }}$ Dec 2020 (submitted in same form $27^{\text {th }}$ Nov 2020)

Protocol v1.0: $3^{\text {rd }}$ Dec 2020

Date recruitment began: $4^{\text {th }}$ Dec 2020

Recruitment complete (estimate): Feb 2021

\section{Background}

Despite the high prevalence of the common mental disorders, anxiety and depression, early in life (1) and their association with poor mental and physical health and socio-economic outcomes throughout the lifespan,(2-4) many young people do not seek or receive help for such disorders.(5-7) Moreover, there is evidence that rates of mental disorders are rising in adolescents and young adults,(8) and even those who have sought help are at high risk of discontinuing their contact with mental health services.(9) Thus, there is increasing interest in the community sector in supporting mental health in young people, however, there is little by way of experimental research in this area.(5)

In the context of COVID-19, economic uncertainty and feelings of isolation brought about by social distancing measures, have meant that mental health and wellbeing have become a major public health 
concern. In the first half of 2020, an epidemiological study demonstrated that 'the prevalence of clinically significant levels of mental distress rose from $18.9 \%$ (95\% Cl 17-8-20.0) in 2018-19 to 27.3\% (26.3-28.2) in April, 2020, one month into UK lockdown.'(10) Some of the greatest increases in scores reflecting the probability of mental disorder were in those aged 18-24-years.

During the first lockdown in the UK many people turned to online content provided by museums and other cultural organisations. We conducted a survey into the potential mental health benefits of online cultural resources on the Ashmolean Museum website. The self-reported mental health benefits of online culture were more marked in regular users of online cultural content and differed with age. People aged 16-24 were less likely to be regular users of online culture, but higher proportions had clinically significant levels of distress, signifying probable anxiety and/or depression. We therefore surmised that targeting by age would be necessary to maximise the benefits of online culture.

We then utilised qualitative research methodologies to investigate the potential mental health benefits of cultural resources in people aged 16-24. We used this research along with co-production methodologies to design and produce an online cultural experience to reduce distress and promote positive mental health in people aged 16-24.

We have designed O-ACE POP (Online Active Community Engagement Proof of Principle Study), a randomised controlled trial, to investigate the efficacy and potential mechanisms of action of an innovative online cultural experience to support mental health and wellbeing in people aged 16-24, compared with a typical museum website.

\section{Methods/design}

The O-ACE POP study is a randomised controlled trial, comparing an online cultural web experience designed to reduce distress and promote positive mental health in people aged 16-24 and a typical museum website. The participant flow chart is shown in Figure 1.

\section{Study Objectives}

1. To compare the effectiveness of the Ways of Being (WoB) web experience with the Ashmolean Museum Website on mood and distress, flourishing and loneliness

2. To explore the potential mechanisms of action of online culture using online cognitive tasks, the Facial Expression Recognition Task (FERT) and the Probabilistic Incentive Learning Task (PILT)

\section{Study Design}

The trial is designed as an online prospective, randomised controlled trial. Eligible participants will be randomised in a 1:1 allocation to WoB or the Ashmolean Website (please see Figure 2).

\section{Ethics}


Online consent procedures will be followed. Ethics approval was provided by the University of Oxford Central University Research Ethics Committee (CUREC), Approval Reference number, R70187/RE007. Any protocol amendments will be communicated to the REC and any changes will be applied after the relevant Ethics approval has been obtained.

\section{Participants and Recruitment}

People aged 16-24 years (16-24 if based in the UK or 18-24 if based overseas) with access to a desktop or laptop computer running a recent browser (Edge or Chrome on Windows, and Safari or Chrome on a Mac) will be eligible for this study.

We will actively recruit people of the target age through social media (Facebook and Instagram), student organizations (e.g. Student Unions) and schools (e.g. via the online publication, The Day) with a link to Participant information and E-consent procedures for those who fulfill inclusion criteria and consent to entering their email address.

Consenting participants will enter a demographic questionnaire including information regarding gender, ethnicity, isolation status, income, relationship status, use of online culture, education, current employment, current and previous mental health and COVID status. Participants will then be emailed a unique ID number and requested to complete a baseline assessment on a computer, including self-report measures and online tasks. They will then be randomised to WoB or the Ashmolean Museum Website.

\section{Interventions}

Ways of Being (WoB) is an online cultural experience designed to reduce distress (symptoms of anxiety and depression) and promote positive mental health in people aged 16-24. The experience centres around the objects and artworks of the Ashmolean Museum in Oxford and partner museums. Based on the findings of qualitative research with people aged 16-24, human centred narratives and viewpoints are a focus of the experience. The experience combines text, audio and aesthetic elements. Each participant will be given a unique ID and we will be able to track the activity of individual participants on WoB.

The Ashmolean Website is a generic museum website owned by the Ashmolean Museum of Art and Archaeology at the University of Oxford. The Ashmolean is the oldest public museum in the UK, and has rich and diverse collections from around the globe, ranging from classical civilisation to the PreRaphaelites and modern art. The website is aimed at general museum visitors, tourists, families, primary, secondary and tertiary learners, teachers, and those with specialist cultural interests. As this is a publicly available Website, we will be unable to track the activity of individual participants.

For both intervention arms, participants will be able to continue with other treatments for anxiety and depression as well as any other activities or interventions that they use to support their mental health. 
Participants will have the option of consenting to text messaging to remind them to complete the intervention and measures over the first part of the trial, including the intense intervention period and the day following. There will also be an email reminder to complete the measures the day following the intense intervention period. In addition, participants will be offered Amazon vouchers as a thank you for their time at certain timepoints (see Participant Information).

\section{Measures}

\section{Mood}

PANAS: The Positive and Negative Affect Schedule (PANAS) is a widely used scale of emotion and comprises 20 items, 10 measuring positive affect (excited, inspired etc) and 10 measuring negative affect (upset, afraid etc), on a Likert Scale of 1 (very slightly) to 5 (extremely). This will be asked with reference to the previous seven days at baseline and follow up, the previous three days at exit and at that precise moment twice daily during the intense intervention period.

K10: The K10 is a well validated self-report measure of distress. (11) It has been widely used as an epidemiological measure to screen for clinically significant depression and anxiety in community samples worldwide. In addition, it has been used as an outcome measure in intervention studies. (12)

\section{Online cognitive tasks}

\section{Facial expression recognition task}

The facial expression recognition task provides an objective measure of emotional bias which is associated with vulnerability to depression. (13) Facial expressions of anger, fear, disgust, sadness, surprise, are presented across different intensity levels (0-100\%, in 10\% steps) and participants are asked to indicate the facial expression that they can see. 250 trials are presented split into separate blocks. Accuracy, reaction time and misclassifications are computed in this task. Low mood is associated with an increased tendency to pick up on negative facial expressions compared to positive ones. (13) This testing and analyses will be supported by the infrastructure of the NIHR Oxford Health Biomedical Research Centre

\section{Probabilistic Incentive Learning Task}

This is an instrumental learning task in which the participant gains or loses points by choosing between two paired images. Participants are required on each trial to choose one of two pairs of symbols. One pair is associated with win outcomes (win 20p or no change) and the other with loss outcomes (lose 20p or no change). Each symbol in the pair correspond to reciprocal probabilities ( 0.7 or 0.3 ) of the associated outcomes occurring. Participants were instructed to pick the symbol they believed was most likely to win (or least likely to lose), with the aim of maximising their monetary pay off. Feedback on the outcome of each trial was given after a choice was made. Participants completed three runs of the task, each with a new set of four symbols. The total amount won, total amount lost, end total, symbol choice and choice 
consistency were recorded. The explicit aim is to maximize points. We chose this task as depression is characterized by deficits in reward-based decision making and therefore changes in the performance of this task may signify changes in the vulnerability to depression.

\section{Secondary outcomes}

\section{Flourishing}

The Flourishing Scale is an 8-item measure of self-perceived success in domains such as self-esteem, relationships, optimism and purpose.(14) It is validated in community samples of young people internationally (15) and provides a single psychological well-being score.

\section{Loneliness}

The UCLA Loneliness Scale is a self-report inventory that uses a Likert-type scale to assess subjective feelings of loneliness. (16) There is a validated three-item version (17) to which we have added a direct measure of loneliness as recommended by the Office of National Statistics for measuring loneliness in those aged 16 and over. (18)

\section{Power analysis and sample size}

We aim to recruit 400 participants. We approximated the statistical power to detect a significant effect of WoB on PANAS scores by assuming an effect size of 0.3 standard deviations of group (WoB vs. Ashmolean Website). With 200 participants in each group, and no pre-intervention differences, a Welch's ttest would have $85 \%$ probability of rejecting the null hypothesis under these parameters.

\section{Randomisation and blinding}

Participants will be randomized using computer generated random sequencing, in blocks of six stratified by gender with an equal allocation ratio. The participants will be allocated by a researcher blinded to all study data except gender during the entire randomisation and allocation procedure. The participants will be aware of which intervention they have been allocated to. The interventions as well as all measures are online and self-report and therefore blinding procedures for care providers and assessors is not relevant.

Both interventions and trial procedures relevant to the first five days of the study have already been trialled on eleven volunteers and detailed feedback taken. This is a low-risk study and we do not anticipate any reasons for unblinding, therefore, a study-specific unblinding procedure has not been put in place. Should the need arise the relevant University of Oxford standard operating procedure will be followed.

\section{Data Management}


The Gorilla database is encrypted. The investigators own the data that has been collected using Gorilla as well as the data. The investigators can generate and access the anonymized data from the Gorilla platform. Data containing personal identifiable information (e.g. email addresses) are stored in a separate database from the anonymised research database; both hosted by Gorilla. Participants who do not complete measures within a reasonable timeframe from allocation will be timed out of the research database. Completed data will be downloaded and safely stored for statistical analysis on the University of Oxford Study drive.

\section{Statistical Analysis Plan}

The main outcome will be the PANAS scale score at each time point (after the baseline and the morning of day 1). These will be regressed on time (pre vs. during and post intensive intervention), a group indicator (WoB vs. the Ashmolean Website), and their interaction. The model will include by-participant random intercepts and effects of time. The Time by Group interaction parameter will indicate the extent to which change in mood (from pre- to post-treatment) is different between the two groups. We plan to conduct two sets of analyses. One to include all those randomised to each intervention and where data is missing, we will use multiple imputation where possible. The other to include all those who used the allocated intervention. We will conduct subgroup analyses based on gender, age group, self-reported anxiety/depression and symptoms at baseline.

\section{Qualitative}

Interview or focus groups will be conducted for each intervention arm. Focus groups will include 5-15 people (purposive sampling) in order to elucidate participant experience, adverse or unintended effects and perspective regarding direction for future research.

\section{Monitoring}

The study will be overseen by a Steering Committee which has had oversight over the trial. Any datarelated issues, should they arise will be discussed with the group of investigators during weekly meetings, in the first instance, and if needed, escalated to the Steering Committee. Access will be provided to authorised representatives from the Sponsor for monitoring and audit purposes if required. The Steering Committee consists of researchers and PPI

We plan to maximise retention with the use of text reminders (for those who consent) and email reminders as well as e-vouchers to thank participants for their time. We will be unable to collect outcome data on participants who have discontinued with the trial.

\section{Discussion}

O-ACE POP has two main goals. The first is to assess the effectiveness of a web-based cultural experience tailored to support mental health in young people compared with the Ashmolean Museum Website, on measures of mood, distress, loneliness and positive mental health. The second is to 
investigate the impact on objective measures (cognitive tasks) predicting emotional vulnerability. Young people identified that human based narratives and other people's viewpoints would have the potential to reduce distress, improve their mental health and enhance feelings of connection with others during the COVID-19 pandemic. This study will allow us to examine whether an intervention based directly on the opinions and experiences of young people and targeted at them, has the potential to work over and above what is already available (without these specific features). This study will allow us to explore who, how and if online cultural experiences are effective for the mental health of young people.

Completing this study in this age range has many important advantages. First, given the trajectory of common mental disorders, this is a critical time point for minimising their impact in terms of disease burden, lifetime distress, illness and social and economic factors such as lost productivity. Second, this is a peak period for development of common mental disorders and, thus, a critical time point for prevention. Third, research in this age range is less likely to be confounded, for example by multiple episodes and medical or psychological interventions.

COVID-19 has been detrimental to the mental health of the population but has provided a unique opportunity to design an innovative approach to supporting mental health in young adults. Findings derived from this study will allow us to evaluate the efficacy of these interventions and will lay the groundwork for the design of studies to further enhance, refine and test online cultural resources for mental health. Moreover, it will allow us to determine whether online experimental studies in the area of community resources for mental health in young people are possible.

\section{Declarations}

\section{Ethics approval and consent to participate}

Online consent procedures will be followed. Ethics approval was provided by the University of Oxford Central University Research Ethics Committee (CUREC), Approval Reference number, R70187/RE007. Any protocol amendments will be communicated to the REC and any changes will be applied after the relevant Ethics approval has been obtained.

\section{Consent for publication}

We can provide a model study consent form on request.

\section{Availability of data and materials}

The authors will have access to the study data. We plan to make the dataset and coding used for the analysis available on the Open Science Framework.

\section{Competing interests}


AKP was supported by grants from the Huo Family Foundation and the Economic and Social Research Council (ES/T008709/1).

ER's role is funded by the NIHR Efficacy and Mechanism Evaluation Programme (16/127/17).

RJSS, MV, HA, CH and JRG report no conflicts of Interest

\section{Funding}

This study is sponsored by the University of Oxford COVID-19 Research Response Fund and supported by the NIHR Oxford Health Biomedical Research Centre, The Westminster Foundation and the Huo Family Foundation. The sponsors did not have a role in designing the study, the collection of the results, in the analysis or interpretation of data, the writing of the manuscript or in the decision to submit for publication. The views expressed are those of the authors and not necessarily those of the NHS, the NIHR or the Department of Health.

\section{Authors' contributions}

Trial design: RJSS, MV, ER, HA, CH, AP and JRG

Trial implementation: RJSS, ER and HA

Manuscript preparation: RJSS, MV, ER, CH, AP and JRG

All authors read and approved the final manuscript.

\section{Acknowledgements}

We would like to thank the following PPI members for their valuable input on the materials used for this trial, Rebecca Woolford, Tharuka Nanomi Arachchige and Louise Chandler

\section{Timeline}

Recruitment and commencement Dec 2020

Expected completion Feb/March 2021

\section{Dissemination Policy}

We plan to publish the results of the study in an academic journal and on the Ashmolean and/or Oxford University Department of Psychiatry websites for members of the public. We will present a summary of key findings in a format that is accessible to a wide audience.

\section{Confidentiality}


Identifiable data (e.g. email addresses) will be stored separately from research data which will only be linked to participant IDs. There will be restricted access to this data on Gorilla to only up to three researchers-members of the team.

Any downloads needed to be made for the purposes of analysing and reporting the results to the Investigators' team will be done on encrypted desktops or laptops and saved immediately in passwordprotected Excel spreadsheets on the restricted-access study folder on the University network drive.

Participant identifiable information will be safely discarded at at the end of the study. If audio-recording is consented to, recordings will be saved on an approved PIN-protected recorder and saved to the restricted access study folder on the University network drive. The audio will be transcribed at a later point. Identifiable information will not be recorded on transcripts. The anonymous transcripts will be linked to participant IDs (PID)s.

\section{References}

1. Kessler RC, Berglund P, Demler O, Jin R, Merikangas KR, Walters EE. Lifetime prevalence and age-ofonset distributions of DSM-IV disorders in the National Comorbidity Survey Replication. Arch Gen Psychiatry. 2005;62(6):593-602. doi:10.1001/archpsyc.62.6.593.

2. Demyttenaere K, Bruffaerts R, Posada-Villa J, Gasquet I, Kovess V, Lepine JP, et al. Prevalence, severity, and unmet need for treatment of mental disorders in the World Health Organization World Mental Health Surveys. JAMA. 2004;291(21):2581-90. doi:10.1001/jama.291.21.2581.

3. Lewinsohn PM, Rohde P, Seeley JR, Klein DN, Gotlib IH. Natural course of adolescent major depressive disorder in a community sample: predictors of recurrence in young adults. Am J Psychiatry. 2000;157(10):1584-91. doi:10.176/appi.ajp.157.10.

4. Nock MK, Hwang I, Sampson N, Kessler RC, Angermeyer M, Beautrais A, et al. Cross-national analysis of the associations among mental disorders and suicidal behavior: findings from the WHO World Mental Health Surveys. PLoS Med. 2009;6(8):e1000123. doi:10.1371/journal.pmed.. Epub 2009 Aug 11.

5. Wolpert M, Dalzell K, Ullman R, Garland L, Cortina M, Hayes D, et al. Strategies not accompanied by a mental health professional to address anxiety and depression in children and young people: a scoping review of range and a systematic review of effectiveness. Lancet Psychiatry. 2019;6(1):4660. doi:10.1016/S2215-0366(18)30465-6. Epub 2018 Dec 3.

6. Rickwood DJ, Deane FP, Wilson CJ. When and how do young people seek professional help for mental health problems? Med J Aust. 2007;187(S7):35-9.

7. Gulliver A, Griffiths KM, Christensen H. Perceived barriers and facilitators to mental health helpseeking in young people: a systematic review. BMC Psychiatry. 2010;10:113.

8. Auerbach RP, Mortier P, Bruffaerts R, Alonso J, Benjet C, Cuijpers P, et al WHO World Mental Health Surveys International College Student Project. Prevalence and distribution of mental disorders. $J$ 
Abnorm Psychol. 2018;127(7):623-38. doi:10.1037/abn0000362. Epub 2018 Sep 13.

9. Kim H, Munson MR, McKay MM. Engagement in Mental Health Treatment Among Adolescents and Young Adults: A Systematic Review. Child Adolesc Soc Work J. 2012;29:241-66.

10. Pierce M, Hope H, Ford T, Hatch S, Hotopf M, John A, et al. Mental health before and during the COVID-19 pandemic: a longitudinal probability sample survey of the UK population. Lancet Psychiatry. 2020;21(20):30308-4.

11. Andrews G, Slade T. Interpreting scores on the Kessler Psychological Distress Scale (K10). Aust N. Z J Public Health. 2001;25(6):494-7.

12. Andrews G, Slade T. Interpreting scores on the Kessler Psychological Distress Scale (K10). Aust N Z J Public Health. 2001;25(6):494-7.

13. Harmer CJ, Cowen PJ, Goodwin GM. Efficacy markers in depression. Journal of psychopharmacology. 2011;25(9):1148-58.

14. Diener W, Tov, Kim-Prieto, Choi, Oishi B-D. New Well-being Measures: Short Scales to Assess Flourishing and Positive and Negative Feelings. Soc Indic Res. 2009;97:143-56.

15. Romano I, Ferro MA, Patte KA, Diener E, Leatherdale ST. Measurement Invariance of the Flourishing Scale among a Large Sample of Canadian Adolescents. Int J Environ Res Public Health. 2020;17(21).

16. Pontinen HM, Swails JA. UCLA Loneliness Scale. In: Zeigler-Hill V, Shackelford TK, editors. Encyclopedia of Personality and Individual Differences. Cham: Springer International Publishing; 2018. pp. 1-3.

17. Hays RD, DiMatteo MR. A short-form measure of loneliness. J Pers Assess. 1987;51(1):69-81.

18. Measuring loneliness. guidance for use of the national indicators on surveys. Office of National Statistics 2018.

\section{Tables}

Table 1: WHO Checklist 


\begin{tabular}{|c|c|}
\hline \multirow{2}{*}{$\begin{array}{l}\text { Primary registry and trial } \\
\text { identifying number }\end{array}$} & https://clinicaltrials.gov \\
\hline & NCT04663594 \\
\hline $\begin{array}{l}\text { Date of registration in } \\
\text { primary registry }\end{array}$ & $11^{\text {th }}$ Dec 2020 \\
\hline $\begin{array}{l}\text { Secondary identifying } \\
\text { numbers }\end{array}$ & $\mathrm{N} / \mathrm{A}$ \\
\hline $\begin{array}{l}\text { Source(s) of monetary } \\
\text { or material support }\end{array}$ & $\begin{array}{l}\text { University of Oxford COVID-19 Research Response Fund, the Westminster } \\
\text { Foundation and the Huo Family Foundation }\end{array}$ \\
\hline Primary sponsor & University of Oxford COVID-19 Research Response Fund \\
\hline Secondary sponsor(s) & The Westminster Foundation \\
\hline $\begin{array}{l}\text { Contact for public } \\
\text { queries }\end{array}$ & Rebecca.sheriff@psych.ox.ac.uk \\
\hline $\begin{array}{l}\text { Contact for scientific } \\
\text { queries }\end{array}$ & Rebecca.sheriff@psych.ox.ac.uk \\
\hline Public title & Online Culture for Mental Health in People Aged 16-24 (O-ACE POP) \\
\hline Scientific title & $\begin{array}{l}\text { A Cultural Experience to support Mental Health in people aged 16-24 } \\
\text { during COVID-19 compared to a typical museum website }\end{array}$ \\
\hline Countries of recruitment & $\begin{array}{l}\text { Online trial (based in the UK but no exclusion of participants from } \\
\text { overseas) }\end{array}$ \\
\hline $\begin{array}{l}\text { Health condition(s) or } \\
\text { problem(s) studied }\end{array}$ & Depression and anxiety \\
\hline \multirow[t]{2}{*}{ Intervention(s) } & The Ashmolean Website \\
\hline & Ways of Being (Web Experience) \\
\hline \multirow{2}{*}{$\begin{array}{l}\text { Key inclusion and } \\
\text { exclusion criteria }\end{array}$} & People aged 16-24 \\
\hline & Exclusion: No access to a laptop or desktop computer \\
\hline Study type & Randomised Controlled Trial \\
\hline Date of first enrolment & $4^{\text {th }} \operatorname{Dec} 2020$ \\
\hline Target sample size & 400 \\
\hline Recruitment status & Ongoing \\
\hline \multirow[t]{2}{*}{ Primary outcome(s) } & Mood (PANAS) \\
\hline & K10 (Kessler Distress Scale) \\
\hline $\begin{array}{l}\text { Key secondary } \\
\text { outcomes }\end{array}$ & $\begin{array}{l}\text { Flourishing, loneliness, Facial Expression Recognition Task, Probabilistic } \\
\text { Incentive Learning Task }\end{array}$ \\
\hline
\end{tabular}




\section{Figures}

Enrollment

Inclusion criteria: $16-24$ years old, computer access

Online Consent

Demographic questionnaire

Baseline measures: current antidepressant, FERT, PILT, mood, anxiety/depression, loneliness, flourishing

Allocation

Intense intervention

Online Culture (WoB) every day for 3 days (and then by choice)

During 3-day intense

Moment-moment mood (twice a day)

Online Culture (Ash) every day for 3 days (and then by choice)

intervention

Day following intense intervention

FERT, PILT, mood, anxiety/depression, loneliness, flourishing

Moment-moment mood (twice a day)

Follow up:

six weeks

FERT, PILT, mood, anxiety/depression, loneliness, flourishing and trial feedback
FERT, PILT, mood, anxiety/depression, loneliness, flourishing

FERT, PILT, mood, anxiety/depression, loneliness, flourishing and trial feedback

FERT: Facial Expression Recognition Task, PILT: Probabilistic Incentive Learning Task, WoB: Ways of Being online cultural experience, Ash: Ashmolean Museum Website

\section{Figure 1}

Participant flow chart 


\begin{tabular}{|c|c|c|c|c|c|c|c|}
\hline \multirow[b]{3}{*}{$\begin{array}{r}\text { TIMEPOINT } \\
\text { (days) } \\
\end{array}$} & \multicolumn{7}{|c|}{ STUDY PERIOD } \\
\hline & \multirow{2}{*}{$\begin{array}{c}\text { Enrolment } \\
-1 \\
\end{array}$} & \multirow{2}{*}{$\begin{array}{c}\text { Allocation } \\
0 \\
\end{array}$} & \multicolumn{4}{|c|}{$\begin{array}{c}\text { Post-allocation (intense } \\
\text { intervention) }\end{array}$} & \multirow{2}{*}{$\begin{array}{l}\text { Close-out } \\
6 \text { weeks }\end{array}$} \\
\hline & & & 1 & 2 & 3 & 4 & \\
\hline \multicolumn{8}{|l|}{ ENROLMENT: } \\
\hline \multirow{3}{*}{$\begin{array}{r}\text { Eligibility screen } \\
\text { Informed } \\
\text { consent } \\
\text { Allocation }\end{array}$} & $x$ & & & & & & \\
\hline & $\mathrm{x}$ & & & & & & \\
\hline & & $x$ & & & & & \\
\hline \multicolumn{8}{|l|}{ INTERVENTIONS } \\
\hline \multicolumn{8}{|l|}{ Ways of Being } \\
\hline $\begin{array}{r}\text { Ashmolean } \\
\text { Website }\end{array}$ & & & $\leftarrow$ & & $\rightarrow$ & & \\
\hline \\
\hline $\begin{array}{c}\text { FERT, PILT, } \\
\text { mood, K10, } \\
\text { loneliness, } \\
\text { flourishing }\end{array}$ & $\mathrm{x}$ & & & & & $\mathrm{X}$ & $\mathrm{x}$ \\
\hline Study feedback & & & & & & & $x$ \\
\hline Twice daily mood & & & $\mathrm{x}$ & $\mathrm{x}$ & $\mathrm{x}$ & & \\
\hline
\end{tabular}

FERT: Fascial Expression Recognition Task, PILT: Probabilistic Incentive Learning Task, K10: Kessler Distress Scale

Figure 2

Schedule of enrolment, interventions, and assessments.

\section{Supplementary Files}

This is a list of supplementary files associated with this preprint. Click to download.

- PLS.docx

- SPIRIT.doc 